\section{Dérégulation de l'expression des gènes à réponse précoce et déficience intellectuelle}

Sarah Boissel ${ }^{1}$, Satoru Hashimoto ${ }^{2}$, Marlène Rio $^{1}$, Mohammed

Zarhrate $^{1}$, Arnold Munnich ${ }^{1}$, Laurence Colleaux ${ }^{1}$, Jean-Marc Egly ${ }^{2}$
${ }^{1}$ Inserm 0781 et département de génétique, fondation Imagine, université Paris Descartes, hôpital Necker-Enfants malades, 149, rue de Sèvres, 75015 Paris, France.

${ }^{2}$ Institut de génétique et de biologie moléculaire et cellulaire, CNRS/Inserm/ université de Strasbourg, BP 163, 67404 Illkirch Cedex, France. laurence.colleaux@inserm.fr

particularité d'être activée de manière rapide et transitoire lorsque la cellule passe de l'état quiescent G0 à la phase Gl du cycle cellulaire, transition qui peut être induite in vitro par l'ajout de sérum dans des cultures cellulaires préalablement privées en sérum.

Pour évaluer les conséquences fonctionnelles de la mutation MED23 R617Q, nous avons donc analysé la réponse au sérum de fibroblastes issus soit d'un patient soit d'un individu contrôle. Alors que la réponse au sérum de $\varepsilon G R I$ était similaire dans les cellules du patient et celles du contrôle, nous avons observé dans les fibroblastes du patient une forte diminution de la stimulation de l'expression de JUN en réponse au sérum et, à l'inverse, une forte augmentation de la stimulation de l'expression de FOS. De plus, la surexpression de la protéine MED23 sauvage dans les cellules du patient restaurait la transactivation des gènes JUN et FOS (mais restait sans effet sur l'expression du gène $\varepsilon G R I$ ), démontrant que le défaut transcriptionnel observé dans les fibroblastes du patient est bien dû à la mutation R6170.

Enfin, par diverses expériences d'immunoprécipitation de chromatine, nous avons démontré que cette dérégulation est la conséquence d'un défaut d'interaction entre la sous-unité MED23 du Mediator et des activateurs spécifiques des deux gènes considérés (TCF4 [T cell factor] dans le cas de JUN et $\varepsilon L K l / 3$ [ $\varepsilon$ twenty-six ETS-like transcription factor 1] dans le cas de FOS). 
Dérégulation de l'expression des gènes à réponse précoce JUN et $F O S$ : une signature moléculaire de la déficience intellectuelle? L'expression des gènes à réponse précoce influe sur le développement et la plasticité cérébrale [6]. Nous avons donc exploré si le défaut transcriptionnel mis en évidence dans les fibroblastes du patient porteur de la mutation MED23 R6170 était retrouvé dans d'autres pathologies avec déficience intellectuelle dues à d'autres défauts du complexe MED. Des mutations du gène MEDI2 ont déjà été associées à deux syndromes de déficience intellectuelle liés à I'X (DILX) : le syndrome d'Opitz-Kaveggia et le syndrome de Lujan. Outre la déficience intellectuelle, ces deux syndromes sont caractérisés par une agénésie du corps calleux, une macrocéphalie, des troubles du comportement, une hypotonie et une dysmorphie $[7,8]$. Nous avons donc analysé l'expression des gènes à réponse précoce $\varepsilon G R 1$, JUN et FOS dans des lignées lymphoblastoïdes dérivées d'un patient atteint du syndrome d'Opitz-Kaveggia et d'un individu contrôle. De manière très intéressante, une altération similaire de l'expression de ces gènes a été observée, suggérant que la dérégulation transcriptionnelle des gènes à réponse précoce JUN et FOS pourrait être une signature moléculaire du déficit cognitif.

La déficience intellectuelle est généralement considérée comme la conséquence d'une anomalie du développement cérébral ou de la plasticité synaptique. II est intéressant de souligner que l'expression des gènes JUN et FOS est activée pendant la période de différenciation neuronale au cours du développement embryonnaire, mais aussi par différents tests de mémoire et d'apprentissage. En effet, chez le rat, Jun est exprimé pendant l'embryogenèse à partir de la neurulation et les études in vitro s'accordent sur son rôle proapoptotique au cours du développement du système nerveux [9]. Par ailleurs, les souris knock-out pour Fos présentent des déficits spécifiques des mémoires associative et spatiale qui ont été corrélés à une réduction de la potentialisation à long terme dans I'hippocampe [10].

Nous proposons donc que la déficience intellectuelle observée chez les patients porteurs d'une mutation dans les gènes MED12 ou MED23 soit due, au moins en partie, à l'altération de l'expression des gènes à réponse précoce dans le cerveau. Cette dérégulation transcriptionnelle pourrait en effet perturber les processus d'apoptose et de différenciation neuronales au cours du développement. Le phénotype pourrait également être dû à une altération de l'expression de ces gènes en réponse à l'activité neuronale, entraînant une dérégulation des cascades de signalisation sous tendant les processus de plasticité synaptique.

\section{Conclusion}

Notre étude montre l'implication d'une nouvelle sous-unité du complexe MED dans une déficience intellectuelle et apporte le premier exemple d'une déficience intellectuelle non syndromique due à un dysfonctionnement de ce complexe. Elle apporte ainsi la démonstration définitive que ce complexe est essentiel au bon fonctionnement cérébral et à la mise en place des fonctions cognitives chez l'homme. Enfin, la caractérisation préliminaire d'une signature moléculaire du déficit cognitif ouvre d'intéressantes perspectives quant à la recherche de molécules thérapeutiques. $\diamond$

Early gene expression dysregulation and intellectual disability

\section{CONFLIT D'INTÉRÊTS}

Les auteurs déclarent n'avoir aucun conflit d'intérêts concernant les données publiées dans cet article.

\section{RÉFÉRENCES}

1. Association AP. DSM-IV: Diagnostic and statistical manual of mental disorders. Washington DC: American Psychiatric Association, 1994.

2. Gecz J, Shoubridge C, Corbett M. The genetic landscape of intellectual disability arising from chromosome X. Trends Genet 2009 ; 25 : 308-16.

3. Hashimoto S, Boissel S, Zarhrate M, et al. MED23 mutation links intellectual disability to dysregulation of immediate early gene expression. Science 2011 ; 333 : 1161-3.

4. Malik S, Roeder RG. The metazoan Mediator co-activator complex as an integrative hub for transcriptional regulation. Nat Rev Genet 2010 ; 11 761-72.

5. Stevens JL, Cantin GT, Wang G, et al. Transcription control by $\varepsilon 1 A$ and MAP kinase pathway via Sur2 mediator subunit. Science $2002 ; 296: 755-8$.

6. Cohen S, Greenberg ME. Communication between the synapse and the nucleus in neuronal development, plasticity, and disease. Annu Rev Cell Dev Biol 2008 ; 24 : 183-209.

7. Risheg H, Graham JM Jr, Clark RD, et al. A recurrent mutation in MED12 leading to R961W causes 0pitzKaveggia syndrome. Nat Genet 2007 ; 39 : 451-3.

8. Schwartz CE, Tarpey PS, Lubs HA, et al. The original Lujan syndrome family has a novel missense mutation (p.N1007S) in the MED12 gene. J Med Genet 2007 ; 44 472-7.

9. Guzowski JF, Setlow B, Wagner EK, McGaugh JL. Experience-dependent gene expression in the rat hippocampus after spatial learning: a comparison of the immediate-early genes Arc, c-fos, and zif268.J Neurosci 2001 ; 21 : 5089-98.

10. Fleischmann A, Hvalby 0 , Jensen V, et al. Impaired long-term memory and NR2A-type NMDA receptordependent synaptic plasticity in mice lacking c-Fos in the CNS. J Neurosci 2003 ; $23: 9116-22$.

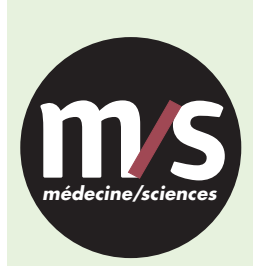

Grâce à $m / s$, vivez en direct les progrès Tarifs d'abonnement $m / s$ - 2012 des sciences biologiques et médicales

Abonnez-vous

à médecine/sciences

Bulletin d'abonnement page 222 dans ce numéro de $\mathrm{m} / \mathrm{s}$

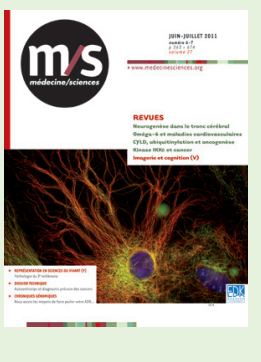

